\title{
Surrogate Model of Flexible Research Platform EnergyPlus Models to Enable Sensitivity Analysis
}

Yanfei Li

Yeonjin Bae

Piljae Im

June 2021 


\title{
DOCUMENT AVAILABILITY
}

Reports produced after January 1, 1996, are generally available free via US Department of Energy (DOE) SciTech Connect.

Website www.osti.gov

Reports produced before January 1, 1996, may be purchased by members of the public from the following source:

\author{
National Technical Information Service \\ 5285 Port Royal Road \\ Springfield, VA 22161 \\ Telephone 703-605-6000 (1-800-553-6847) \\ TDD 703-487-4639 \\ Fax 703-605-6900 \\ E-mail info@ntis.gov \\ Website http://classic.ntis.gov/
}

Reports are available to DOE employees, DOE contractors, Energy Technology Data Exchange representatives, and International Nuclear Information System representatives from the following source:

Office of Scientific and Technical Information

PO Box 62

Oak Ridge, TN 37831

Telephone 865-576-8401

Fax 865-576-5728

E-mail reports@osti.gov

Website https://www.osti.gov/

This report was prepared as an account of work sponsored by an agency of the United States Government. Neither the United States Government nor any agency thereof, nor any of their employees, makes any warranty, express or implied, or assumes any legal liability or responsibility for the accuracy, completeness, or usefulness of any information, apparatus, product, or process disclosed, or represents that its use would not infringe privately owned rights. Reference herein to any specific commercial product, process, or service by trade name, trademark, manufacturer, or otherwise, does not necessarily constitute or imply its endorsement, recommendation, or favoring by the United States Government or any agency thereof. The views and opinions of authors expressed herein do not necessarily state or reflect those of the United States Government or any agency thereof. 
Electrification and Energy Infrastructures Division Grid Interactives Control Group

\title{
SURROGATE MODEL OF FLEXIBLE RESEARCH PLATFORM ENERGYPLUS MODELS TO ENABLE SENSITIVITY ANALYSIS
}

\author{
Yanfei Li \\ Yeonjin Bae \\ Piljae Im
}

June 2021

\author{
Prepared by \\ OAK RIDGE NATIONAL LABORATORY \\ Oak Ridge, TN 37831-6283 \\ managed by \\ UT-BATTELLE LLC \\ for the \\ US DEPARTMENT OF ENERGY \\ under contract DE-AC05-00OR22725
}





\begin{abstract}
This letter report describes the surrogate models developed from the EnergyPlus model of Oak Ridge National Laboratory's Flexible Research Platform. Two data-driven black-box models were developed, and the outputs of the surrogate models were compared with the EnergyPlus model. The two models developed are a multilayer perceptron deep learning model, and a long short-term memory (LSTM) neural network model. The three factors for selecting the black-box models are scalability, computation time, and accuracy. A total of 107 input variables were the dominant variables in determining the outputs of building energy consumptions and thermal comfort. A total of 54 output variables were identified as the prediction targets, including the system- and zone-level outputs.
\end{abstract}

The large set of the simulation cases were generated by integrating sensor errors into an emulator based on EnergyPlus and Python EMS, which includes advanced control sequences from ASHRAE Guideline 36-2018: High-Performance Sequences of Operation. The surrogate models were developed based on a set of large-scale simulation runs (i.e., 4,000 runs) on a cloud platform. The comparison analysis shows that the two black-box models had good accuracy for predicting new outputs for sensitivity analysis using the root mean square error metric.

As a next step, the developed surrogate models will be used to perform sensitivity analysis for different sensor impacts (e.g., sensor types, sensor locations).

\title{
1. INTRODUCTION
}

The Sensor Impact Evaluation and Verification project is a 3-year, 3-laboratory collaborative project. The overarching project goal is to develop a framework that allows quantitative evaluation of the impact of sensors on building HVAC control, fault detection and diagnostics, and consequently, building energy efficiency and occupant thermal comfort. To achieve the goal in FY 2021, the Oak Ridge National Laboratory team identified variables of interest and developed an emulator that is a custom module using Python and the Flexible Research Platform (FRP) EnergyPlus Python plugin coupled with heuristic controllers for one use case, which is room temperature control. The developed emulator enables evaluating the sensor impact with various parameters related to building HVAC systems, control, and sensors. However, to quantitatively assess the effect of parameters on building performance, uncertainty and sensitivity analysis needs to be performed. This analysis requires an extensive number of simulations, making the use of the developed emulators for this purpose difficult. To enable this analysis, the team developed surrogate models, and this report presents the method to develop the surrogate models from the EnergyPlus model of Oak Ridge National Laboratory's FRP, and its results.

\section{CLOUD COMPUTING}

\subsection{SENSORS}

Based on extensive literature reviews, 34 sensors were identified. They are typical sensors to operate rooftop unit (RTU) and variable air volume (VAV) systems in small to medium office buildings. The sensors were prioritized based on indoor air dry-bulb temperature, which potentially affects energy efficiency and occupant thermal comfort significantly.

The identified sensors are frequently found in commercial buildings. They are listed in Table 1. 
Table 1. Comprehensive sensor list.

\begin{tabular}{|c|c|c|c|c|c|}
\hline Location & Measurement & Priority & Location & Measurement & Priority \\
\hline Room & Temperature & 1 & RTU & OA CO $_{2}$ & 4 \\
\hline Room & Humidity & 3 & RTU & OA flow rate & 3 \\
\hline Room & $\mathrm{CO}_{2}$ & 4 & RTU & SA temperature & 1 \\
\hline Room & Lighting condition & 5 & RTU & SA humidity & 3 \\
\hline Room & Occupancy & 5 & RTU & SA CO $_{2}$ & 4 \\
\hline VAV box & SA temperature & 1 & RTU & SA flow rate & 3 \\
\hline VAV box & SA humidity & 3 & RTU & RA temperature & 2 \\
\hline VAV box & SA flow rate & 1 & RTU & RA humidity & 3 \\
\hline Main duct & Static pressure & 2 & RTU & RA CO & 4 \\
\hline Exhaust fan & EA temperature & 4 & RTU & RA flow rate & 3 \\
\hline Exhaust fan & EA humidity & 4 & RTU & MA temperature & 2 \\
\hline Exhaust fan & EA flow rate & 4 & RTU & MA humidity & 3 \\
\hline Exhaust fan & EA CO & & 4 \\
\hline Other & Plug load & 4 & RTU & MA CO & \\
\hline Other & Lighting load & 5 & RTU & Refrigerant temperature & 5 \\
\hline RTU & OA temperature & 1 & RTU & Refrigerant pressure & 5 \\
\hline RTU & OA humidity & 3 & RTU & Refrigerant flow rate & 5 \\
\hline
\end{tabular}

$\mathrm{SA}=$ supply air; $\mathrm{EA}=$ exhaust air; $\mathrm{OA}=$ outdoor air; $\mathrm{RA}=$ return air; $\mathrm{MA}=$ mixing air

Based on the FRP building and HVAC system, five sensor types were selected for the following reasons: the zone air temperature is the most important variable to control to meet the set points; the zone air temperature is directly impacted by the VAV box supply air (SA) temperature and SA flow rate from the control perspective; and RTU system-level operation also directly impacts the VAV operations; RTU outdoor air (OA) temperature and supply air temperature are significant in determining the system-level energy consumption. The sensor types are listed in Table 2.

Table 2. Selected sensor list.

\begin{tabular}{|c|c|c|}
\hline Location & Measurement & Priority \\
\hline Room & Temperature & 1 \\
\hline VAV box & SA temperature & 1 \\
\hline VAV box & SA flow rate & 1 \\
\hline RTU & OA temperature & 1 \\
\hline RTU & SA temperature & 1 \\
\hline
\end{tabular}

\subsection{SENSORS ERRORS (INPUTS)}

The sensor error comprises two components: bias error and precision error. Figure 1 (right) shows the demonstration of bias error and precision error. For a sensor, there is an ideal reading (or true reading) at a time step, as shown in the black line of Figure 1. The bias error is the bias of average sensor readings from the ideal readings, as shown in green dotted line of Figure 1. It is a system error. The precision error is the random bias from the average sensor readings, as shown in the blue lines of Figure 1. 

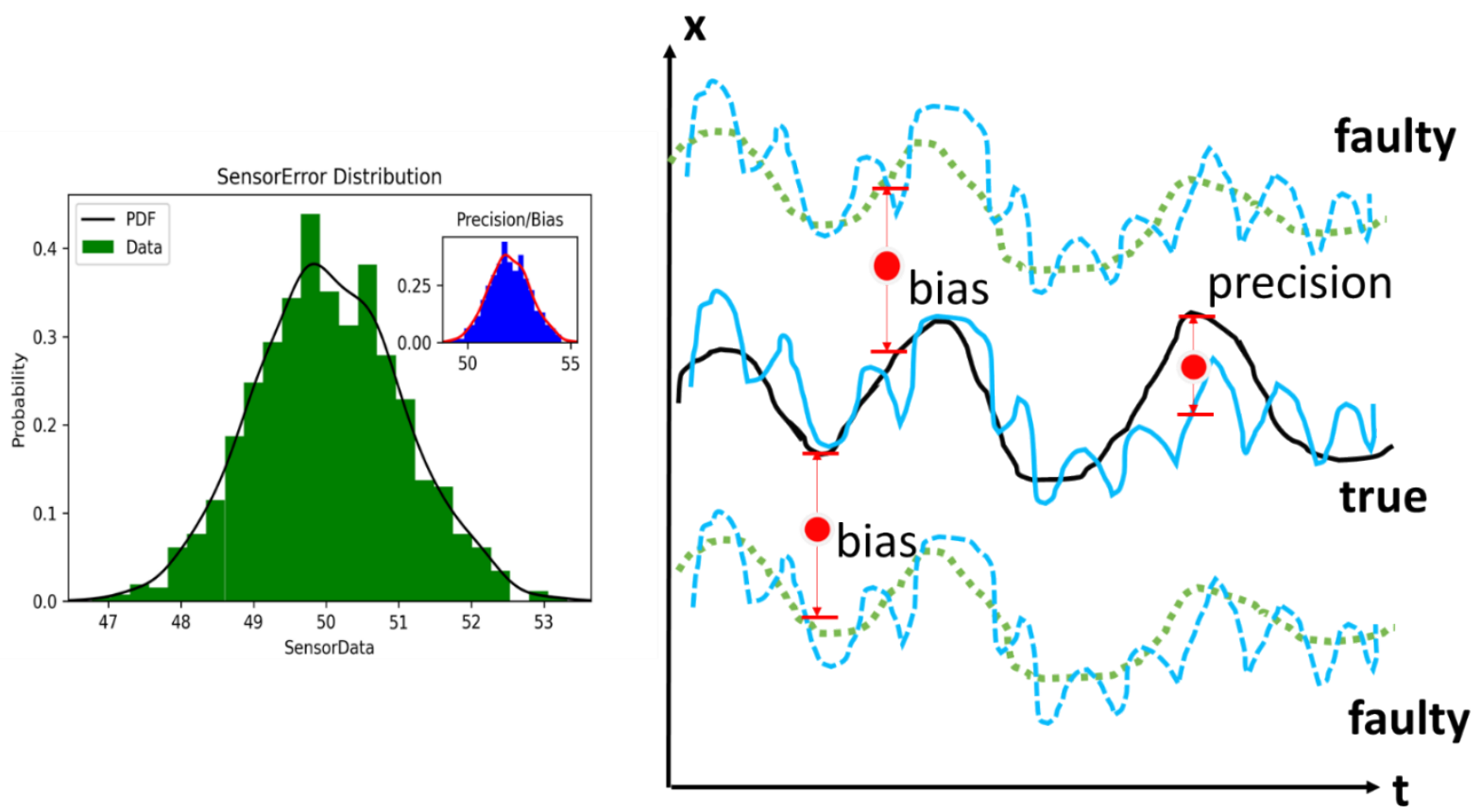

Figure 1. Sensor Error Diagram (note: PDF means probability density function)

Both the bias error and precision error follow normal distributions, as shown in Figure 1 (left).

Equations (1)-(3) describe the sensor errors:

$$
\begin{gathered}
\operatorname{Err}_{\text {bias }}=\text { normal }_{\text {distribution }}\left(0, \sigma_{\text {bias }}\right), \\
\operatorname{Err}_{\text {precision }}=\text { normal }_{\text {distribution }}\left(0, \sigma_{\text {precision }}\right), \\
\text { Sensor }_{\text {readings }}=\text { Sensor }_{\text {idealreadings }}+\operatorname{Err}_{\text {bias }}+\operatorname{Err}_{\text {precision }},
\end{gathered}
$$

where

$\sigma_{\text {bias }}$ is the standard deviation of bias error, and $\sigma_{\text {precision }}$ is the standard deviation of precision.

The sensor errors were injected based on the principles of above given equations.

\subsection{LARGE-SCALE SIMULATION}

The large-scale simulation was based on a commercial cloud platform, Microsoft Azure. A total of 4,000 cases were simulated on the cloud. The inputs were the sensor errors injected for the five selected sensors for the FRP building emulator, as shown in Table 3. Because of EnergyPlus internal programming limits, injecting larger sensor errors leads to simulation crash. The sensor error standard deviations were based on multiple trials. The thresholds were based on engineering experience and domain knowledge, and also on actual RTU- and zone-level sensor ideal readings. The outputs were the target variables for energy consumption and thermal comfort, such as fan electricity consumption and VAV box reheat coil energy. 
Table 3. Selected sensor error standard deviation.

\begin{tabular}{|c|c|c|r|c|}
\hline Location & Measurement & Bias & Precision & Note \\
\hline Room & Temperature & 1 & 0.1 & ${ }^{\circ} \mathrm{C}$ \\
\hline VAV box & SA temperature & 1 & 0.1 & ${ }^{\circ} \mathrm{C}$ \\
\hline VAV box & SA flow rate & 0.001 & 0.0001 & $\mathrm{~m}^{3} / \mathrm{s}$ \\
\hline RTU & OA temperature & 1 & 0.1 & ${ }^{\circ} \mathrm{C}$ \\
\hline RTU & SA temperature & 1 & 0.1 & ${ }^{\circ} \mathrm{C}$ \\
\hline
\end{tabular}

The basic diagram is shown in Figure 2. The basic workflow is as follows:

1. A Python script is developed to generate 4,000 simulation input files (IDF files). Each IDF file is associated a Python class of sensor errors through Python EMS. During the simulation, at each time step, a new sensor error (including bias and precision) is injected to the ideal sensor readings from EnergyPlus.

2. After 4,000 cases are generated, they are uploaded to the Azure cloud platform.

3. In the Azure cloud platform, a bash script selects the appropriate virtual machine configurations (e.g., memory, hard drive, as shown in Table 4) and number of virtual machines. The team's subscription includes 300 nodes (virtual machines).

4. The Azure cloud provides a job scheduler, which automatically distributes all 4,000 cases through 300 nodes.

5. The simulation marches automatically until all cases are accomplished.

6. Finally, all the results are downloaded to set up the data sets (inputs and outputs) to create the blackbox models.

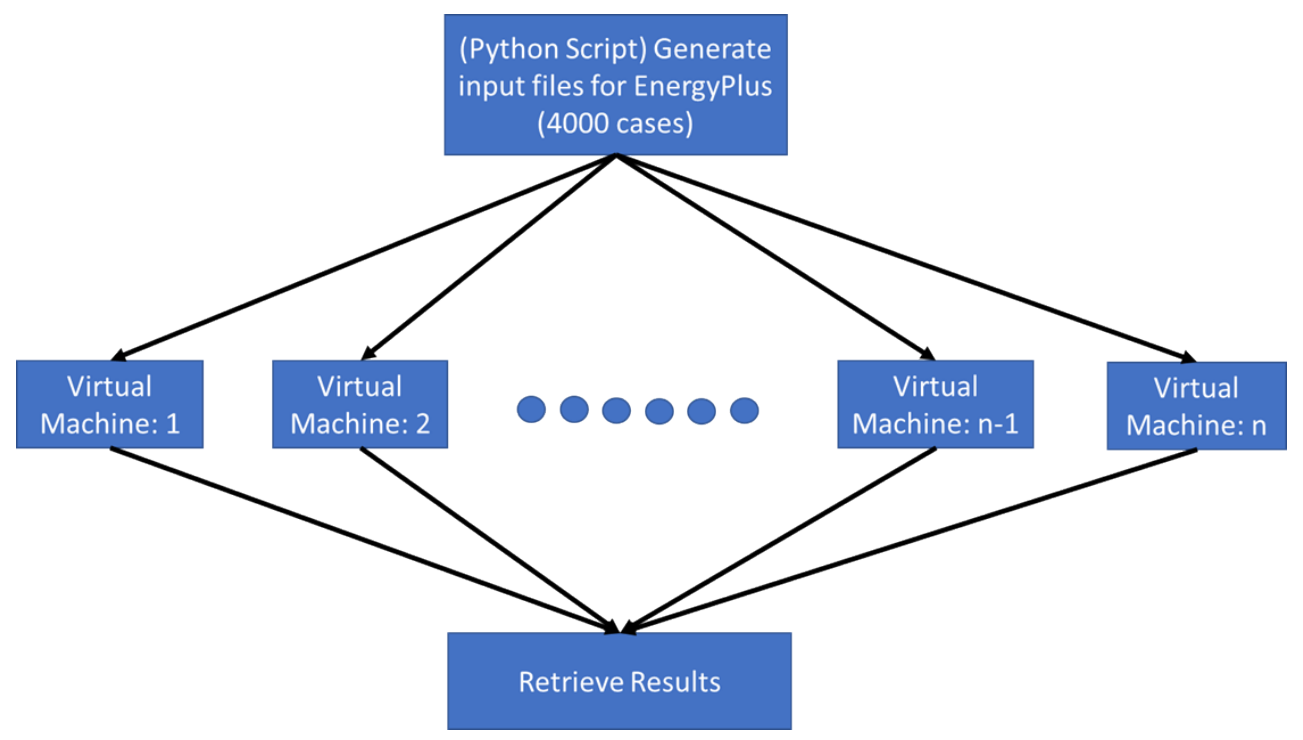

Figure 2. Large-scale simulation information flow.

The configuration for the cloud is shown in Table 4. 
Table 4. Cloud configurations.

\begin{tabular}{|c|c|c|c|c|c|}
\hline Machine type & CPUs/cores & Memory & $\begin{array}{c}\text { Storage/ } \\
\text { SSD }\end{array}$ & $\begin{array}{c}\text { Total } \\
\text { nodes }\end{array}$ & $\begin{array}{c}\text { Time } \\
\text { executed }\end{array}$ \\
\hline Standard_D16d_v4 & 16 & $64 \mathrm{~GB}$ & $600 \mathrm{~GB}$ & 300 & $9 \mathrm{~h}$ \\
\hline
\end{tabular}

The diagram for each case is shown in Figure 3. The sensor list was preselected based on previous studies. The sensor errors were sampled through normal distribution for each time step. The sensor readings from EnergyPlus used the sensor errors to form a hacked sensor readings. The hacked sensor readings were used as inputs to control sequences to calculate new set points. These new set points were used to control the performance of buildings. Ultimately, the energy consumption and thermal comfort were different from using the ideal sensor readings.



Figure 3. FRP EnergyPlus emulator.

\section{SURROGATE MODELS}

\subsection{INPUTS AND OUTPUTS}

The purpose of the surrogate model was to establish the mapping relationship between input and output variables. The input variables were based on the FRP EnergyPlus models. A detailed list of variables is provided in Table 5 . 
Table 5. Input variables.

\begin{tabular}{|c|c|}
\hline Variable name & Quantity \\
\hline OAT & 1 \\
\hline OAT relative humidity & 1 \\
\hline OA pressure & 1 \\
\hline Wind speed & 1 \\
\hline Wind direction & 1 \\
\hline Horizontal infrared radiation rate & 1 \\
\hline Diffuse solar radiation rate & 1 \\
\hline Direct solar radiation rate & 1 \\
\hline Lighting energy & 1 \\
\hline Internal heat gains: equipment & 1 \\
\hline People Activity & 1 \\
\hline SensorBias: AHU OAT & 1 \\
\hline SensorPrecision: AHU OAT & 1 \\
\hline SensorTotalError: AHU OAT & 1 \\
\hline SensorBias: AHU SAT & 1 \\
\hline SensorPrecision: AHU SAT & 1 \\
\hline SensorTotalError: AHU SAT & 1 \\
\hline SensorBias: zone VAV SAF & 10 \\
\hline SensorPrecision: zone VAV SAF & 10 \\
\hline SensorTotalError: zone VAV SAF & 10 \\
\hline SensorBias: zone VAV SAT & 10 \\
\hline SensorPrecision: zone VAV SAT & 10 \\
\hline SensorTotalError: zone VAV SAT & 10 \\
\hline SensorBias: zone air temperature & 10 \\
\hline SensorPrecision: zone air temperature & 10 \\
\hline SensorTotalError: zone air temperature & 10 \\
\hline Total & 107 \\
\hline
\end{tabular}

(Note: $\mathrm{OAT}=$ outdoor air temperature, $\mathrm{SAT}=$ supply air temperature, $\mathrm{SAF}=$ supply air flow rate)

Similarly, the output variables were also based on FRP EnergyPlus models. A detailed list of output variables is provided in Table 6 . 
Table 6. Output variables.

\begin{tabular}{|c|c|c|}
\hline Variable name & Quantity & Note \\
\hline Fan electricity rate & 1 & \\
\hline Main cooling coil sensible cooling rate & 1 & \\
\hline Main cooling coil electricity rate & 1 & \\
\hline Main heating coil heating rate & 1 & \\
\hline Zone air sensible heating rate & 10 & \\
\hline Zone air sensible cooling rate & 10 & \\
\hline Zone air temperature & 10 & \\
\hline Zone predicted percentage dissatisfied & 10 & \\
\hline VAV box reheat energy & 10 & \\
\hline RTU cooling temperature set point & 1 & Optional \\
\hline RTU heating temperature set point & 1 & Optional \\
\hline VAV box supply airflow rate set point & 10 & Optional \\
\hline Total & 66 & \\
\hline
\end{tabular}

\subsection{TRAINING AND TESTING}

The whole data set was divided into a training data set ( $80 \%$ of total) and a validation data set ( $20 \%$ of total). The training data set was used to learn the weights of input variables to output variables. The validation data set was used to test the accuracy of the surrogate model prediction from the emulator output variables. The dataset were all shuffled to avoid the input data internal impacts. The root mean squared error (RMSE) is used to quantify the modeling accuracy, which is given as below:

$$
R M S E=\sqrt{\frac{\sum_{1}^{N}\left(y_{i}-\hat{y}_{i}\right)^{2}}{N}},
$$

where $R M S E$ is the root mean square error, $y_{i}$ is the emulator output variable, $\hat{y}_{i}$ is the surrogate model output variable, and $N$ is the total number of variables in prediction.

\subsection{MULTILAYER PERCEPTRON MODEL}

\subsubsection{Principle}

The multilayer perceptron (MLP) functioned as a black-box model for regression with the desired input and output variables, as shown in Figure 4. The structure diagram is shown in Figure 5. For black-box models, the purpose is to find the mapping relationship between inputs and outputs. The MLP model is a technique to find such mappings (or "weights") or each input variables using a gradient descent method. It has input layers (with all input variables), hidden layers, and output layers (with all output variables). The gradient descent method is a mature mathematical method widely used in machine learning. The details are not discussed here. 




Figure 4. MLP principle.

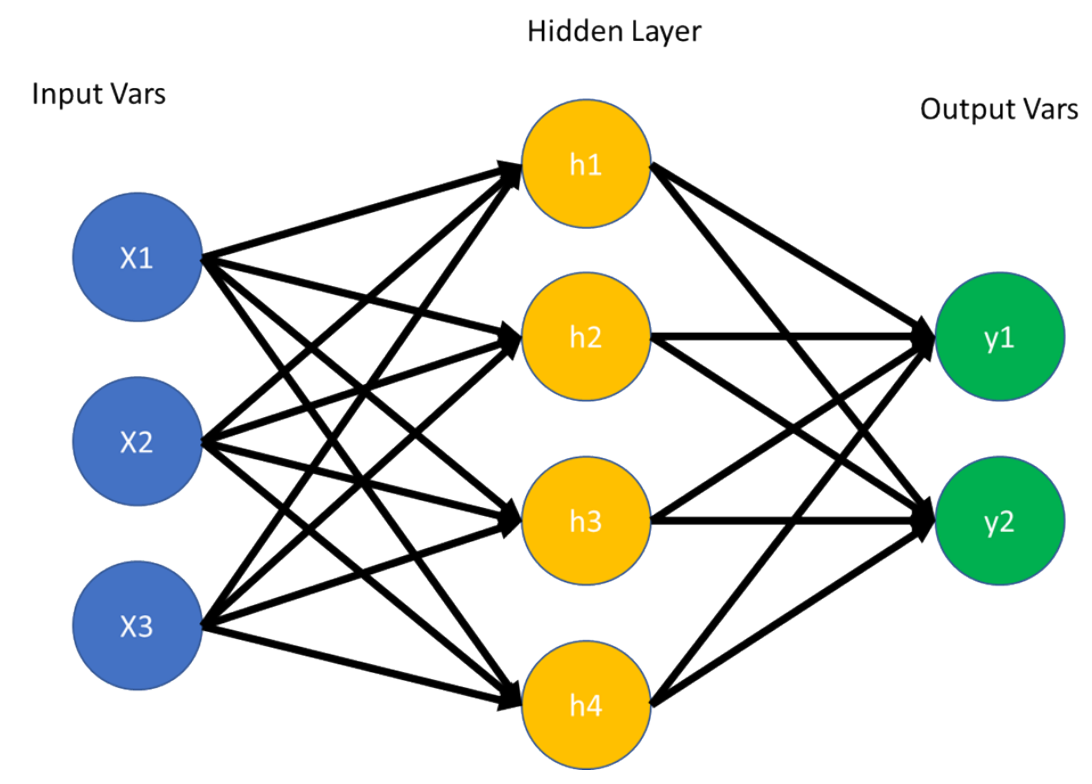

Figure 5. MLP structure.

In Figure 5, the input variables $(\mathrm{x} 1, \mathrm{x} 2, \mathrm{x} 3)$ and output variables (e.g. y1, y2) are associated together, to learn the weights (coefficients) based on large training dataset. The hidden layer (e.g. h1, h2, h3, h4) acts as intermediate variables.

\subsubsection{Results}

The results for the MLP model are shown in Figure 6 (RTU system-level energy consumptions) and Figure 7 (zone-level energy consumptions and thermal comfort). The RMSE metric was used to quantify the model accuracy. 

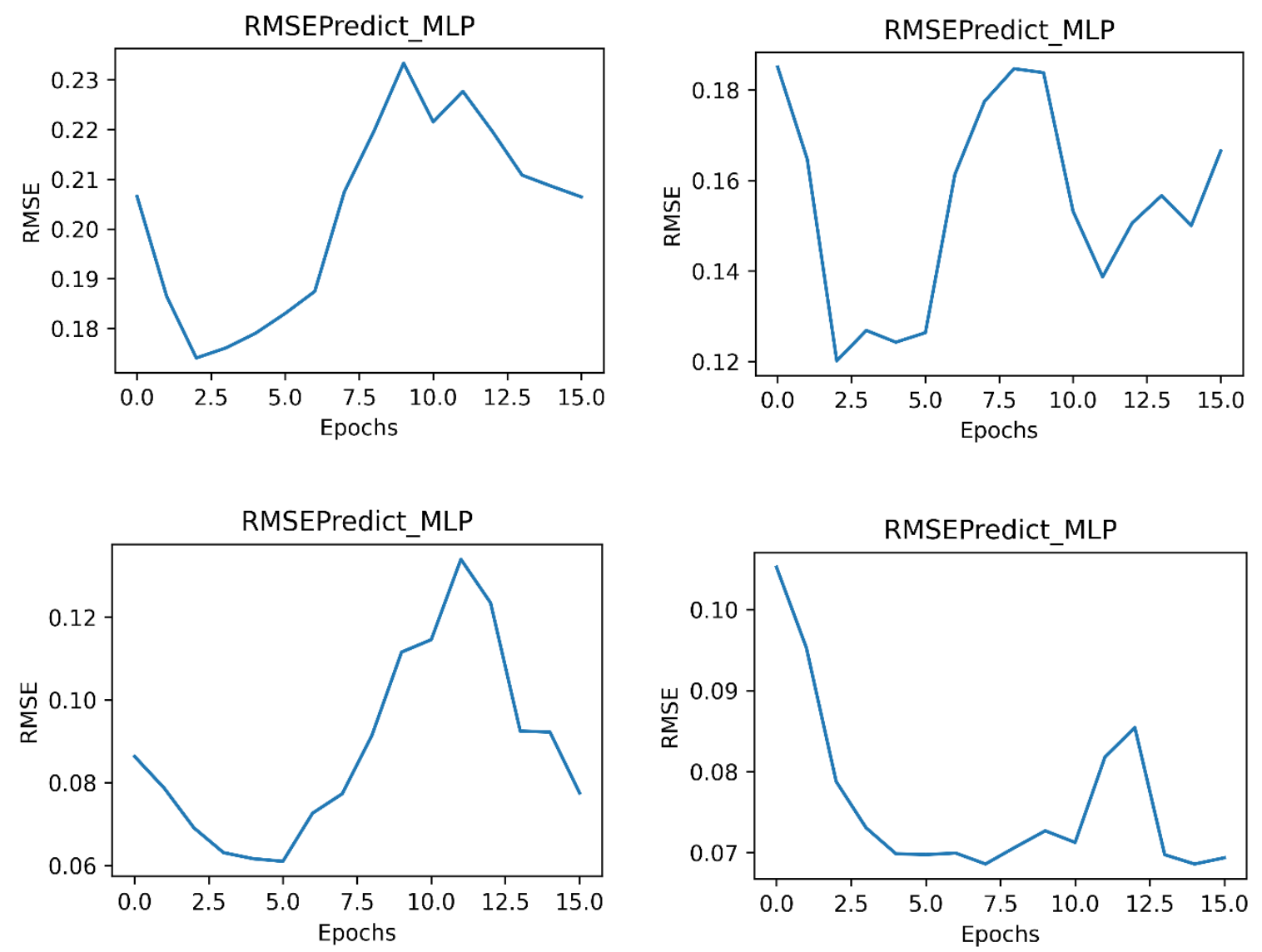

Figure 6. RTU system-level energy outputs (MLP). Top left: fan electricity consumption; top right: cooling system electricity consumption; bottom left: cooling system cooling energy; bottom right: heating system heating energy. 

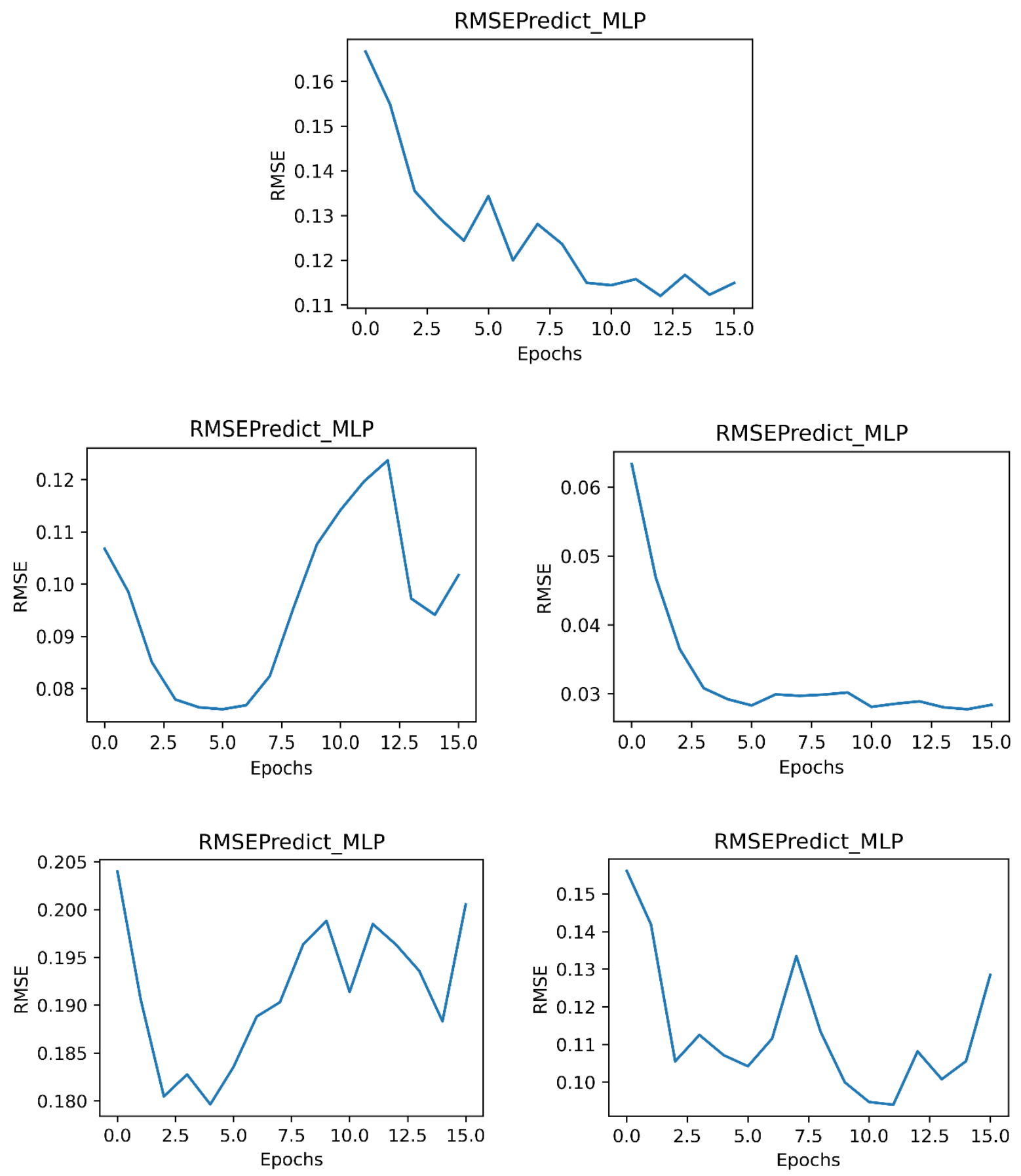

Figure 7. Zone-level energy outputs (zone 102). Top: zone sensible heating energy; 2nd row left: zone VAV box reheat coil energy; 2nd row right: zone cooling energy; 3rd row left: zone predicted percentage dissatisfied; 3rd row right: zone temperature.

Figure 6 demonstrates the RTU system-level energy items based on the MLP model. The energy items are fan electricity consumption, cooling system electricity consumption, cooling system cooling energy, and heating system heating energy. The RMSE metric shows that they had good agreement for training and testing of the simulation data set. 
Figure 7 demonstrates the zone-level energy items based on the MLP model. The energy items are zone sensible heating energy, zone VAV box reheat coil heating energy, zone sensible cooling energy, zone predicted percentage dissatisfied, and zone temperature. The RMSE metric shows that a good agreement for the MLP models.

\subsection{LONG SHORT-TERM MEMORY MODEL}

\subsubsection{Principle}

Similar to the MLP model, the long short-term memory (LSTM) model is a neural network model suitable for time-series forecasting. For building energy simulations, the results are time-series variables. The thermal state of buildings at previous time steps has certain impacts on the later time steps. With LSTM, the main purpose is to find the mapping of inputs and outputs (Figure 8). Figure 9 shows that the input variables were transformed into multiple routes as a way of including previous states' impacts. The detailed mathematics are not included here because the goal was to use LSTM to make a black-box model.

Time-series data: $1 \mathrm{~min}$ resolution

Time-series output: $1 \mathrm{~min}$ resolution

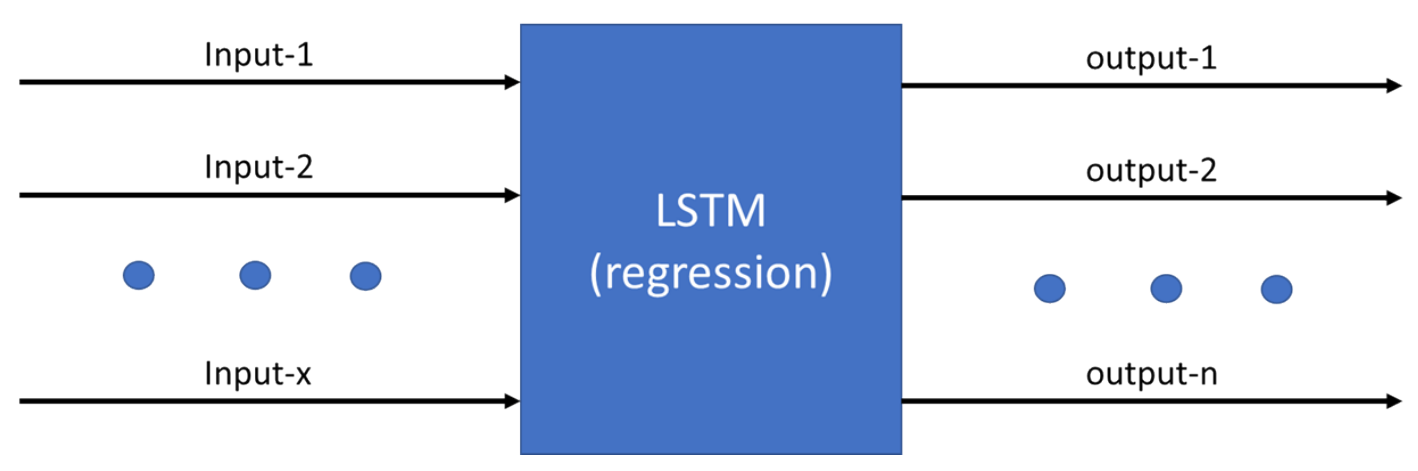

Figure 8. LSTM principle. 


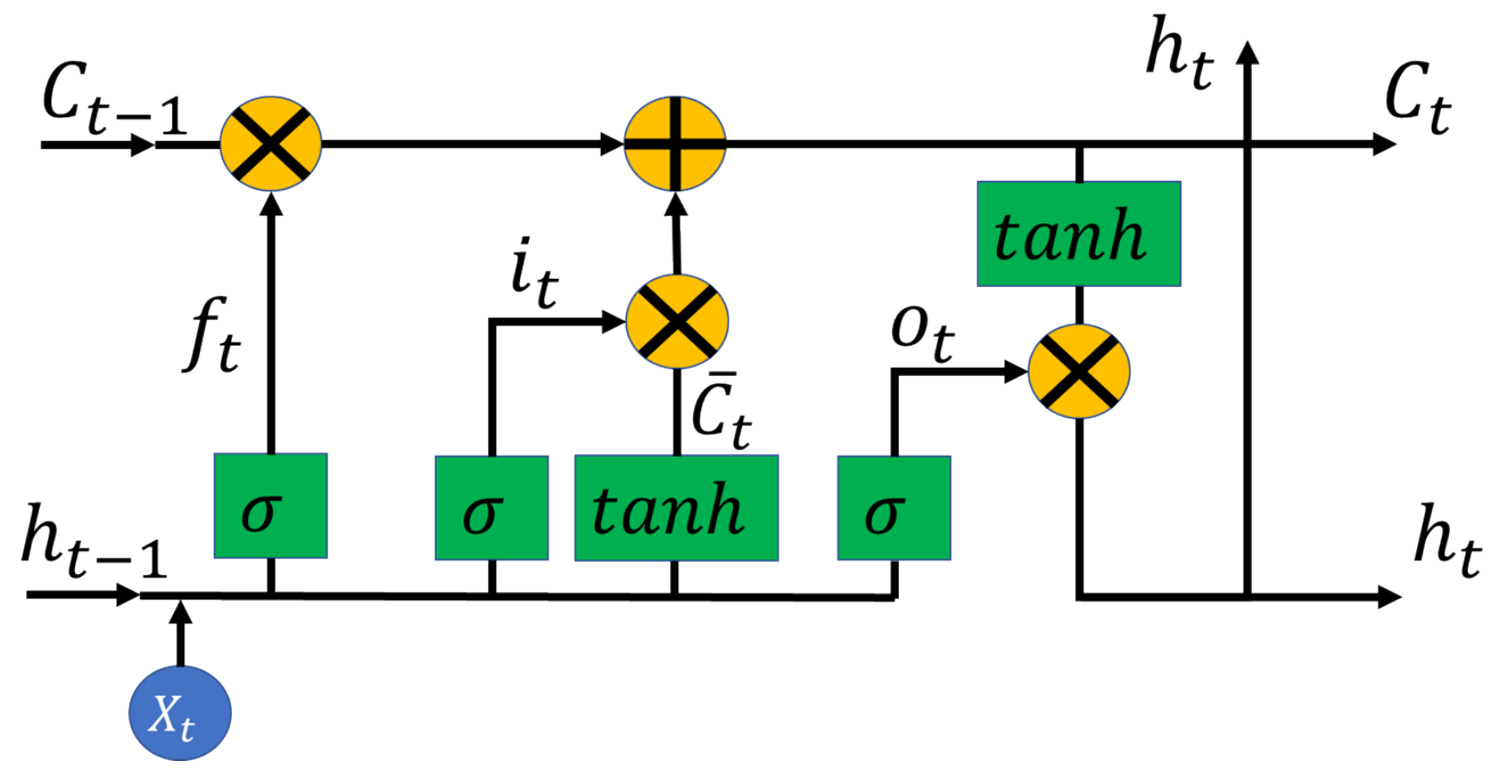

Figure 9. LSTM cell structure.

Similarly, the LSTM model was used as a black-box for regression, which is shown in Figure 8 . The detailed structure of the LSTM model is shown in Figure 9. The LSTM structure has many variations, and only the common one was used. The mathematical principles are not included here, because it is only used as a tool for data-driven purpose.

\subsubsection{Results}

The results for the LSTM model are shown in Figure 10 (RTU system-level energy consumptions) and Figure 11 (zone-level energy consumptions and thermal comfort). The RMSE metric was used to quantify the model accuracy. 

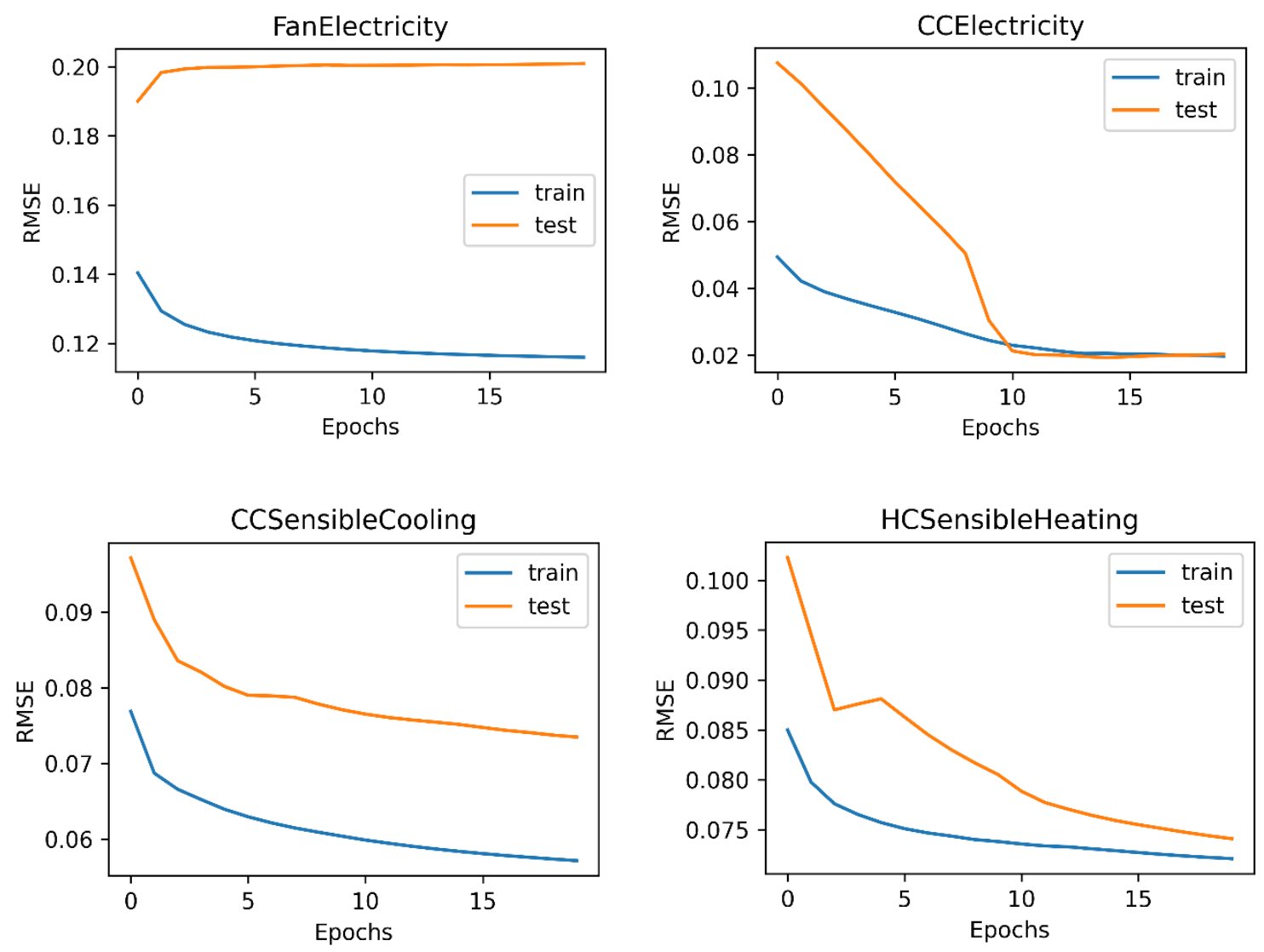

Figure 10. RTU system-level energy outputs. Top left: fan electricity consumption; top right: cooling system electricity consumption; bottom left: cooling system cooling energy; bottom right: heating system heating energy. 

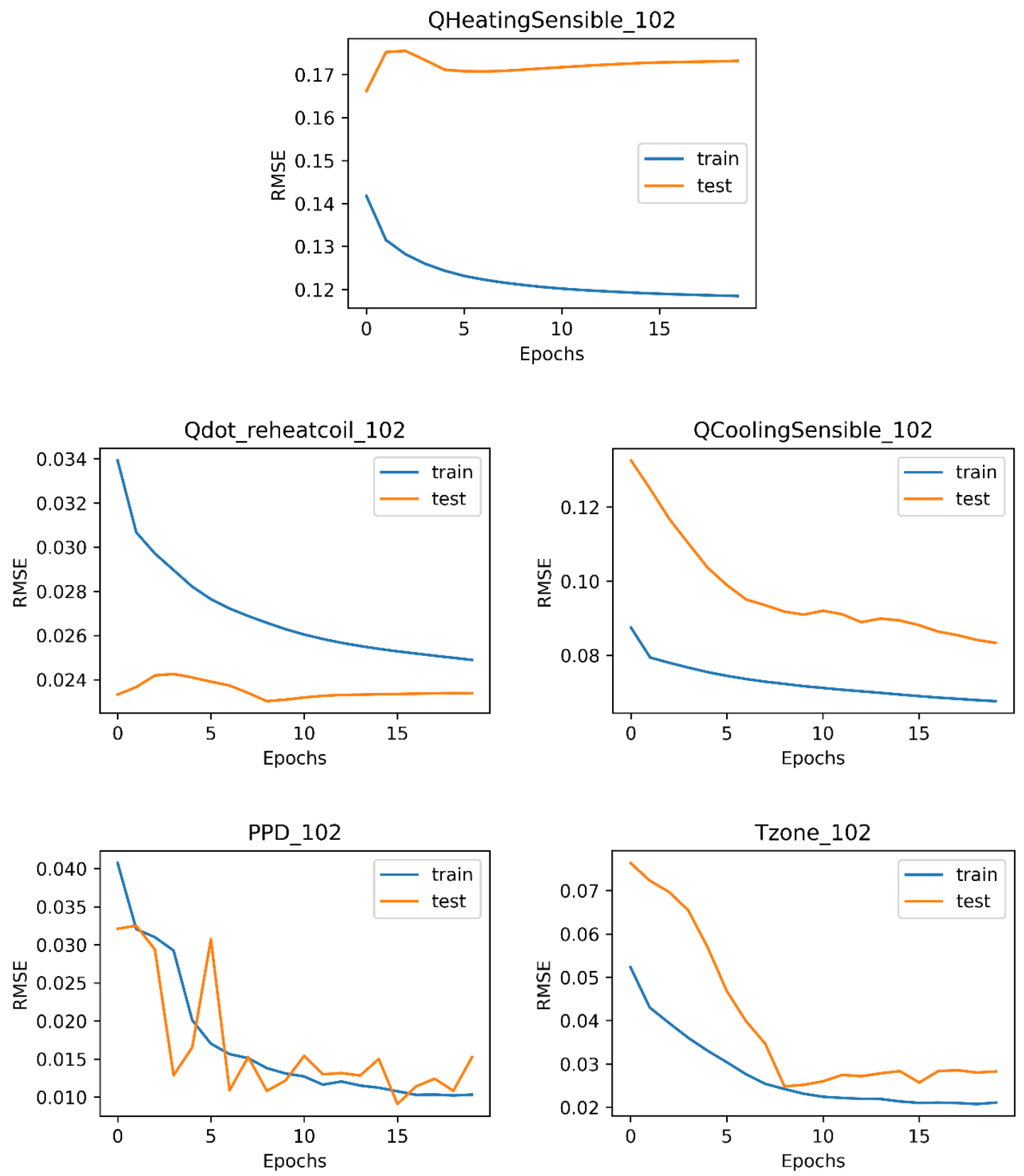

Figure 11. Zone-level energy outputs. Top: zone sensible heating energy; 2nd row left: zone VAV box reheat coil energy; 2nd row right: zone cooling energy; 3rd row left: zone predicted percentage dissatisfied; 3rd row right: zone temperature.

Figure 10 demonstrates the RTU system-level energy prediction based on the LSTM model. The energy items are fan electricity consumption, RTU cooling electricity consumption, cooling energy, and heating energy. The RMSE metric shows that the model was sufficient for prediction in sensitivity analysis.

Figure 11 demonstrates the zone-level energy prediction based on the LSTM model. The energy items are zone sensible heating energy, VAV box reheat coil energy, zone sensible cooling energy, zone predicted percentage dissatisfied, and zone temperature. The RMSE metric shows that the LSTM model had better accuracy than the MLP model. 


\section{CONCLUSIONS}

This milestone investigated the surrogate models for sensitivity analysis. Various steps were involved for the surrogate models. Typical sensors were selected for the FRP building. Sensor errors were identified accordingly. An emulator was established with the sensor types, sensor errors, and EnergyPlus model. The advanced control sequence was integrated. Large-scale simulations were conducted (4,000 simulation cases) on the cloud. After downloading the simulation results, 107 input variables and 54 output variables were selected. The mapping relationship of the variables was obtained through machine learning techniques. Two surrogate models were proposed: MLP and LSTM. Three factors in selecting these surrogate models were scalability, computation time, and accuracy. Based on the simulation data set, the two surrogate models were trained, and they showed good agreement with simulation results. The LSTM model was slightly more accurate than the MLP model.

Next, the surrogate models will be used for sensitivity analysis. The proposed workload is to establish a sensitivity analysis-oriented framework using the surrogate models, perform sensitivity and uncertainty analysis based on 4,000 simulation cases, and perturb sensor locations - and conduct multiple sets of large-scale simulations - for sensitivity analysis. 


(n)

